\title{
OSCILlations IN THE YARN TENSION DURING THE YARN UNWINDING FROM PACKAGES
}

\author{
PraceK, S.; Sluga, F. \& Mozina, K.
}

Abstract: In textile production, the yarn is being withdrawn from cross-wound packages in warping and weft insertion. During unwinding, there appear forces in the yarn which are approximately proportional to the square of the unwinding velocity. The production process requires as large warping and weaving speeds as possible, therefore it is necessary to improve our understanding of the cross-wound package unwinding and to find the necessary modifications of the yarn unwinding process. In addition to empirical tests, it has proved useful to study yarn unwinding by mathematical modelling and computer simulations. We state the eguations of motion that describe the unwinding yarn and develop a mathematical model which permits to simulate the process of unwinding.

Key words: yarn unwinding, equations of motion, ballon theory, unwinding simulations, oscillations of stress in yarn
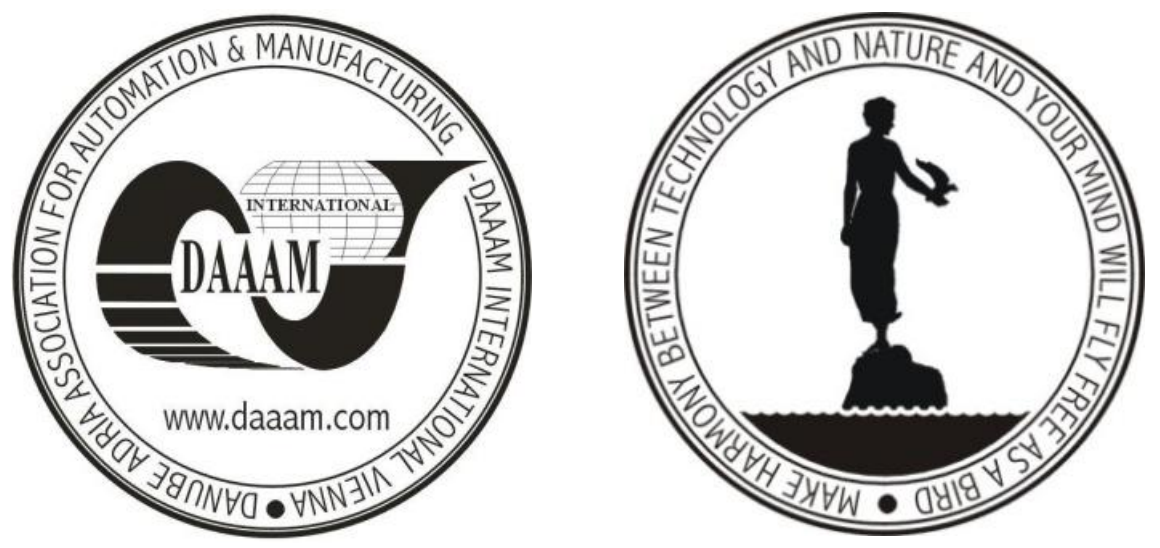

Authors' data: Doc. Dr. Sc. Pracek, S[tanislav]; Univ. Prof. Dr. Sc. Sluga, F[ranci]; M.Sc. Mozina, K[lemen], University of ljubljana, NTF, Department of textile, Snezniska 5, 1000 Ljubljana, Slovenija, stane.pracek@ntf.uni-lj.si, franci.sluga@ntf.uni-lj.si, klemen.mozina@ntf.uni-lj.si

This Publication has to be referred as: Pracek, S[tanislav]; Sluga, F[ranci] \& Mozina, K[lemen] (2011). Oscillations in the Yarn Tension During the Yarn Unwinding from Packages, Chapter 35 in DAAAM International Scientific Book 2011, pp. 433-444, B. Katalinic (Ed.), Published by DAAAM International, ISBN 978-3-901509-84-1, ISSN 1726-9687, Vienna, Austria

DOI: $10.2507 /$ daaam.scibook.2011.35 
Pracek, S.; Sluga, F. \& Mozina,K.: Oscillations in the Yarn Tension During the Y...

\section{Introduction}

Oscillations in the yarn tension during the yarn unwinding from stationary packages have a direct influence on the quality of the fabric. The characteristics of the unwinding process are thus important for production of high quality garments and should therefore be optimized. The theory of yarn dynamics during unwinding was developed as early as in 1950's (Padfield, 1958) and is still an active area of research (Praček, 2002) . The theory was recently compared with experimental results with considerable success (Barr \& Catling, 1976; Kothari \& Leaf, 1979 ; Fraser \& Ghosh \& Batra, 1992 ; Fraser, 1992; Clark \& Fraser \& Sharma \& Rahn, 1998; Clark \& Fraser\&Stump, 2001). This demonstrates that theoretical modelling of yarn unwinding process can be used to simulate and then optimize the parameters of the package construction and the unwinding process. In our previous work (Praček, 2007) we showed how such a mathematical model can be devised and we described some general characteristics of the unwinding process. In this work we review the results of our extensive simulations and show how the unwinding properties of a package can be improved by using alternating layers of parallely wound and cross wound layers.



y

Fig.1. The yarn is being withdrawn with velocity $\mathrm{V}$ through an eyelet, where we also fix the origin $\mathrm{O}$ of our coordinate system (Fig.1). The yarn is rotating aroun the $\mathrm{z}$ axis with an angular velocity $\omega$. At the lift-off point Dv the yarn lifts from the package and forms a balloon. At the unwinding point Od the yarn starts to slide on the surface of the package. Angle $\phi$ is the winding angle of the yarn on the package

We now consider the equation of motion of yarn (Praček, 2002):

$$
\rho\left(D^{2} r+2 \omega \times D r+\omega \times(\omega \times r)+\dot{\omega} \times r\right)=\frac{\partial}{\partial s}\left(T \frac{\partial r}{\partial s}\right)+f
$$

Here $\rho$ is the mass per unit length of the yarn, $\mathbf{r}$ is the radius vector to a given point on the yarn, $\mathrm{D}$ is the comoving time derivative operator, $\mathrm{T}$ is the yarn tension and $\mathbf{f}$ is the density of external forces acting on the yarn. In the part of the yarn that forms the balloon $\mathbf{f}$ is the density of the air drag force(Roberson \& Crowe, 1980): 


$$
f=-\frac{1}{2} c_{u} \rho d\left|v_{n}\right| v_{n}
$$

where $c_{u}$ is the air drag coefficient, $d$ is the diameter of the yarn and $v_{n}$ is the normal component of the yarn velocity. In the part of the yarn that slides on the package (between the unwinding and lift-off point) $f$ is the density of forces with which package resists the motion of the yarn. It consists of the normal force of the package and the force of friction:

$$
f=n e_{r}-\mu n \frac{v}{|v|}
$$

where $\mathrm{n}$ is the strength of normal force, $e_{r}$ is the radial unit vector and $\mu$ is the coefficient of friction.It is worthwile to cast the equation of motion in the dimensionless form. This consists of finding "natural" units in which to express all the quantities appearing in the equation. A number of constants will remain in the reduced equation and these dimensionless parameters have the most pronounced effect on the yarn dynamics.

We express all distances in units of package radius:

$$
\bar{r}=\frac{r}{c}, \bar{z}=\frac{z}{c}, \bar{s}=\frac{s}{c}
$$

time is expressed in units of period of balloon rotation:

$$
\bar{t}=\frac{t}{\tau}=\omega t
$$

velocitis are expressed in units of unwinding speeed:

$$
\bar{v}=\frac{v}{V}, \bar{v}_{n}=\frac{v_{n}}{V}
$$

and finally we find the following suitable combinations of quantities for forces and tension:

$$
\begin{aligned}
& \bar{f}=\frac{f c}{\rho V^{2}} \\
& \bar{n}=\frac{n c}{\rho V^{2}} \\
& \bar{T}=\frac{T}{\rho V^{2}}
\end{aligned}
$$


Pracek, S.; Sluga, F. \& Mozina,K.: Oscillations in the Yarn Tension During the Y...

When we rewrite the equation of motion with these dimensionless quantities we obtain

$$
\bar{D}^{2} \bar{r}+2 \Omega \times \bar{D} \bar{r}+\Omega \times(\Omega \times \bar{r})+\Omega \frac{\partial}{\overline{\partial t}} \times \bar{r}=\frac{\partial}{\partial \bar{s}}\left(\overline{\bar{T}} \frac{\bar{r}}{\overline{\partial s}}\right)+f
$$

Only one parameter remains in this equation (without taking into account the external force term). It is the dimensionless angular velocity $\Omega$ :

$$
\Omega=\frac{c \omega}{V}
$$

This is the single most important parameter in our model. We will show later on that we can make many important conclusions if we determine how $\Omega$ changes with time as the yarn is being unwound.

There are two additional dimensionless parameters: $\mu$, the coefficient of friction between yarn and package, and the coefficient of air resistance $p_{0}$ :

$$
p_{0}=\frac{16 c D_{n}}{\rho}
$$

These two parameters are approximately constant during the unwinding, so they are less important.

The coefficient of friction depends on the quality of the yarn and on the surface properties of the package. The quality of the surface is comparable for forward and backward unwinding direction, so that the coefficient of friction remains approximately constant.

\section{Model of unwinding proces}

In packages of general shape (cylindrical or conical) the relation between the angular velocity of the yarn during unwinding $\omega$, the unwinding speed $V$ and the package radius $c$ at the lift-off point, where the yarn lifts off from the package surface, is (Praček, 2007):

$$
\omega=\frac{V}{c} \frac{\cos \phi}{1-\cos \alpha \sin \phi}
$$

Here $\phi$ is the winding angle and $\alpha$ is the apex angle of the conical package (for cylindrical packages we put $\alpha=0$ ).

The lift-off point moves up and down the package during unwinding. The exact time dependence of $\omega$ is defined by the shape of the package (described by a suitable time 
dependant $c$ ) and the type of winding (described by a suitable time dependant $\phi$ ). In order to perform simulations, we additionally need a relation between the unwinding velocity and the yarn tension. The tension is largest in the eyelet through which the yarn is being pulled (Fraser, 1992) and this is a convenient point to perform experimental measurements of the tension. In the following when we use the word 'tension', we refer to the tension at the eyelet and we denote it by T.

From the simplest theory of the yarn unwinding which doesn't take into account the air drag nor the Coriolis force, we know that the tension is proportional to the angular velocity squared:

$$
T_{0} \propto \omega^{2}
$$

This expression is only approximately true. A better estimate could be obtained from the full equation of motion for the yarn, but no simple analytic expression can be obtained in this case. In addition, a balloon limiter is usually used during the unwinding. This device limits the radius of the balloon, thereby reducing the centrifugal force on the yarn in the balloon and consequently the tension is lower as well. For this reason we performed experimental measurements to determine the relation between the tension and the angular velocity. We measured tension for parallely wound cylindrical packages of different dimensions and for different unwinding velocities. For such packages we have $\alpha=0$ and $\phi \sim 0$, so that $\omega=V / c$.

We performed a series of measurements for different unwinding velocities $V$ and package radii $c$. We thus obtain the tension for a range of angular velocities and we used a linear interpolation between these values to obtain the relation for the whole interval of values of interest (Fig. 2). If during the simulation, a value of $\omega$ is outside this interval, we can use linear extrapolation for values above the highest $\omega$ measured and a quadratic extrapolation for values below the lowest $\omega$ measured, in accordance with Eq. (12).

In building this model we make a few assumptions:

1. The length of the yarn in the balloon has no effect on the tension.

2. We neglect the residual tension of yarn in the package, which is related to the stiffness of the winding.

3. The winding angle and the number of the threads are approximately constant in the number of the layers whose unwinding will be simulated.

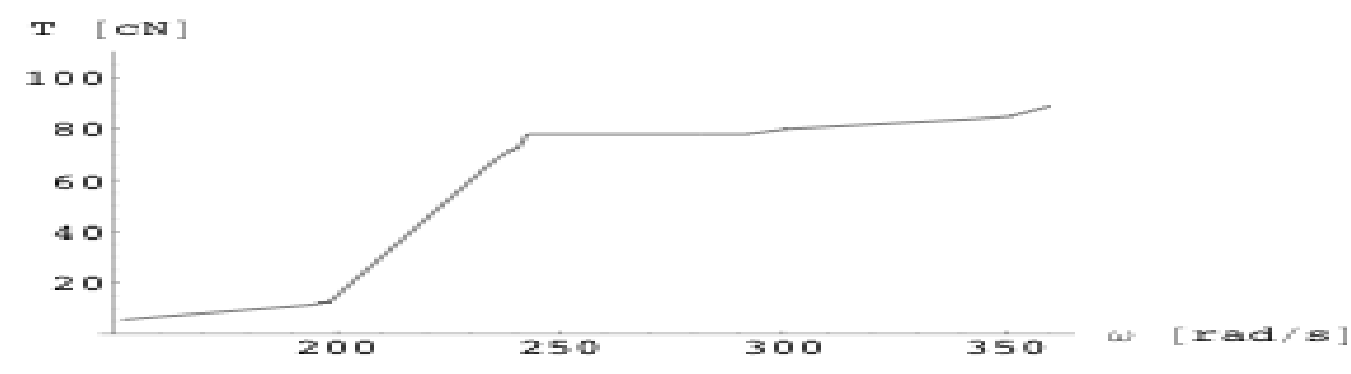

Fig. 2: Dependence of tension $\mathrm{T}$ on angular velocity $\omega$ of the yarn. 
Dependence of $\mathrm{T}$ on $\omega$, based on experimental results for cylindrical parallely wound package with cotton yarn 41.6 tex

A few comments about the dependence of $\mathrm{T}$ on $\omega$ (Fig. 2) are in order. At low $\omega$, up to approximately $\omega=240 \mathrm{rad} / \mathrm{s}$, the tension increases approximately quadratically, which is to be expected from the formula in Eq. (12). After the limiting value of $\omega=240 \mathrm{rad} / \mathrm{s}$, the tension is bounded and increases only slightly. This is a direct consequence of using the balloon limiter in the measurements: at the limiting value of the angular velocity the radius of the balloon becomes large enough that it is limited by the balloon limiter. The centrifugal forces then no longer increase with $\omega$ and the tension can only grow due to the yarn friction with the surface of the limiter and other less controllable reasons. Since similar balloon limiters are also used in the actual industrial process, it is advantageous to take them into account in our model.

\section{Results of unwinding simulation}

We now present the results of the simulations. We investigated the effects of different unwinding velocities $V$, package radii $c$, the winding angles $\phi$ and, for conical packages, the apex angle $\alpha$.

\subsection{Cilindrical packages}

In Fig. 3 we show the tension during the unwinding at $V=1500 \mathrm{~m} / \mathrm{min}$ and at radius $c=100 \mathrm{~mm}$ for different winding angles. For densely parallely wound packages $(\phi \sim 0)$ the tension is constant, as expected, since in parallely wound packages the angular velocity is approximately constant. In cross wound packages the velocity is time dependant. When the yarn unwinds backwards the angular velocity is higher as when the yarn unwinds forwards, which follows from Eq. (11). Since the tension strongly depends on the angular velocity, we obtain oscillations in the yarn tension. The abrupt change in the tension occurs when the lift-off points reaches an edge of the package. Such changes strain the yarn and can lead to damage. In the most extreme case, the yarn can break. In designing new package types it is therefore necessary to limit both the maximum tension in the yarn and the amplitude of oscillations during unwinding.

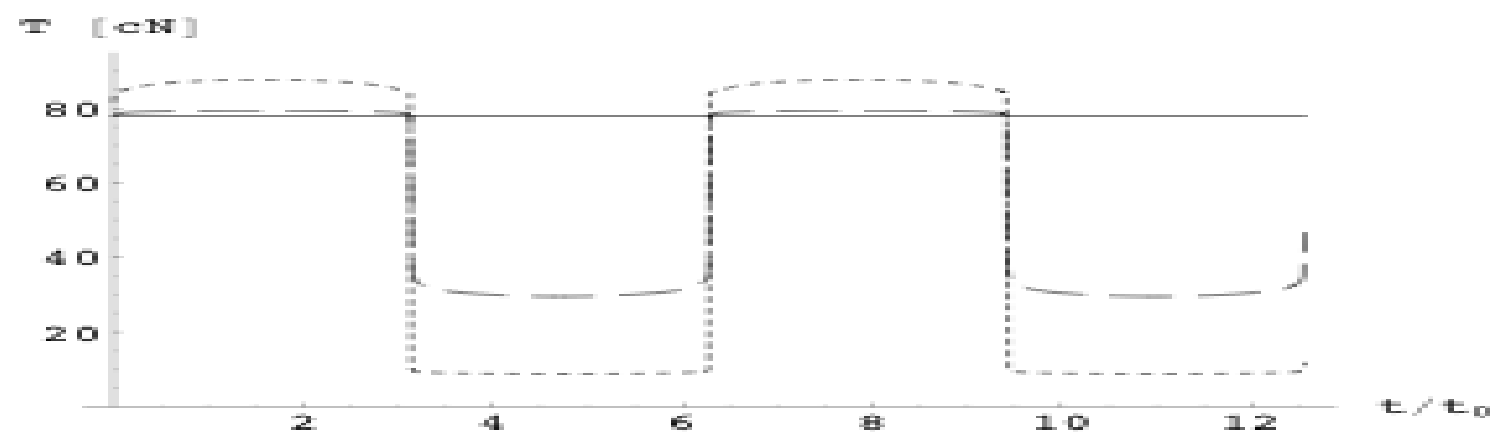

Fig. 3: Tension in the yarn during unwinding at $V=1500 \mathrm{~m} / \mathrm{min}$ and at radius $c=100$ $\mathrm{mm}$ for different winding angles: $\phi=0^{\circ}$ (solid line), $\phi=10^{\circ}$ (dashed line) and $\phi=20^{\circ}$ (dotted line) 
In Fig. 4 we show unwinding from a similar package, but at higher unwinding velocity $V=2000 \mathrm{~m} / \mathrm{min}$. Tensions are in this case very high. Unwinding from such packages might not be possible.

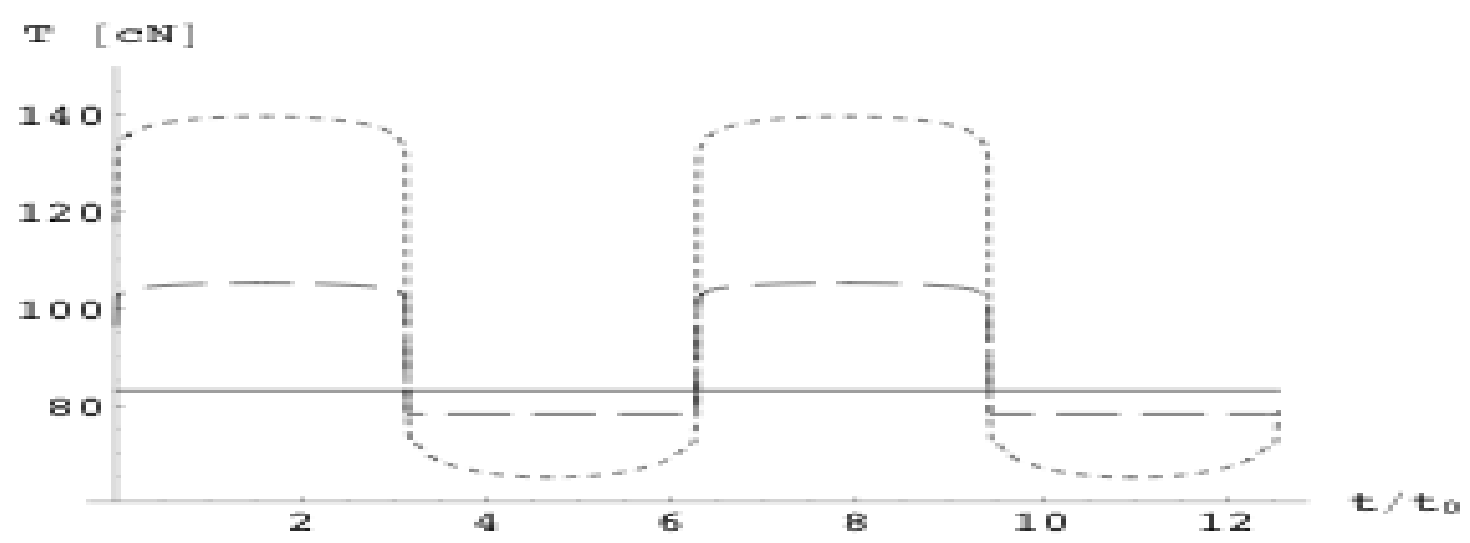

Fig. 4: Tension in the yarn during unwinding at $V=2000 \mathrm{~m} / \mathrm{min}$ and at radius $c=100$ mm for different winding angles: $\phi=0^{\circ}$ (solid line), $\phi=10^{\circ}$ (dashed line) and $\phi=20^{\circ}$ (dotted line)

In Fig. 5 we show a 3D plot of tension as a function of time and package radius. For large radius packages the tension is significantly reduced. Therefore it is still possible to unwind at very high unwinding velocities from packages with sufficiently large smallest radius (as determined by the radius of the tube on which the yarn is wound). We have determined that the minimum radius should be around $150 \mathrm{~mm}$ in order to keep the tension below $50 \mathrm{cN}$.

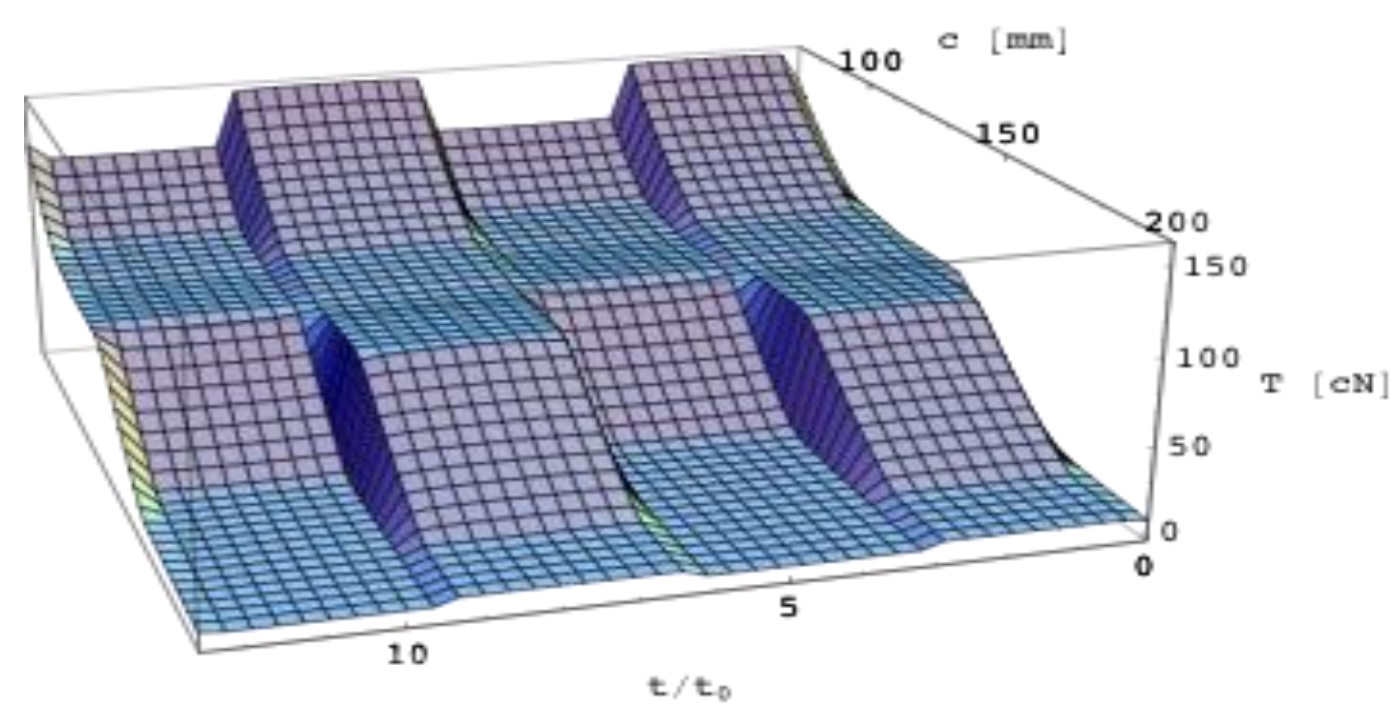

Fig. 5. Tension in the yarn as a function of package radius. $V=2000 \mathrm{~m} / \mathrm{min}, \phi=5^{\circ}$

This limiting value of $50 \mathrm{cN}$ is chosen so that the largest deformation in the yarn is in the elastic regime, i.e. the tension in the yarn should be below $10 \%$ of the breaking tension. The values were determined experimentally. During unwinding tests we achieved up to $17 \%$ of the breaking tension without affecting the 
Pracek, S.; Sluga, F. \& Mozina,K.: Oscillations in the Yarn Tension During the Y...

mechanical properties of the yarn: the breaking tension of the yarn after unwinding was not reduced. We determined the breaking tension to be $4.5 \mathrm{~N}=450 \mathrm{cN}$, from which follows the estimate of $50 \mathrm{cN}$ for the maximum allowable tension.

The oscillations of the tension are related to the oscillations in the angular velocity of the yarn. The amplitude of the angular velocity oscillations can be obtained as

$$
\begin{aligned}
& \Delta \omega=\omega_{\max }-\omega_{\min }=\frac{V}{c} \frac{\cos \phi}{1-\sin \phi}-\frac{V}{c} \frac{\cos (-\phi)}{1-\sin (-\phi)} \\
& =\frac{2 V}{c} \tan \phi .
\end{aligned}
$$

In the region of interest, i.e. for $\phi<25^{\circ}$, we can approximate $\tan \phi \sim \phi$, so we have

$$
\Delta \omega \approx \frac{2 V \phi}{c} .
$$

According to this expression, the amplitude of angular velocity oscillations is approximately proportional to the unwinding velocity and the winding angle and inversely proportional to the package radius. From Fig. 2 we can then read off the maximum and minimum of tension and plot the difference.

In Fig. 6 we plot the amplitude of oscillations of the tension as a function of the winding angle and the package radius for a constant unwinding velocity $V=2000$ $\mathrm{m} / \mathrm{min}$. Oscillations become very large for increasing value of the winding angle. For packages of small radius, $c=70 \mathrm{~mm}$, unwinding is only safe for winding angle below $\phi=5^{\circ}$.

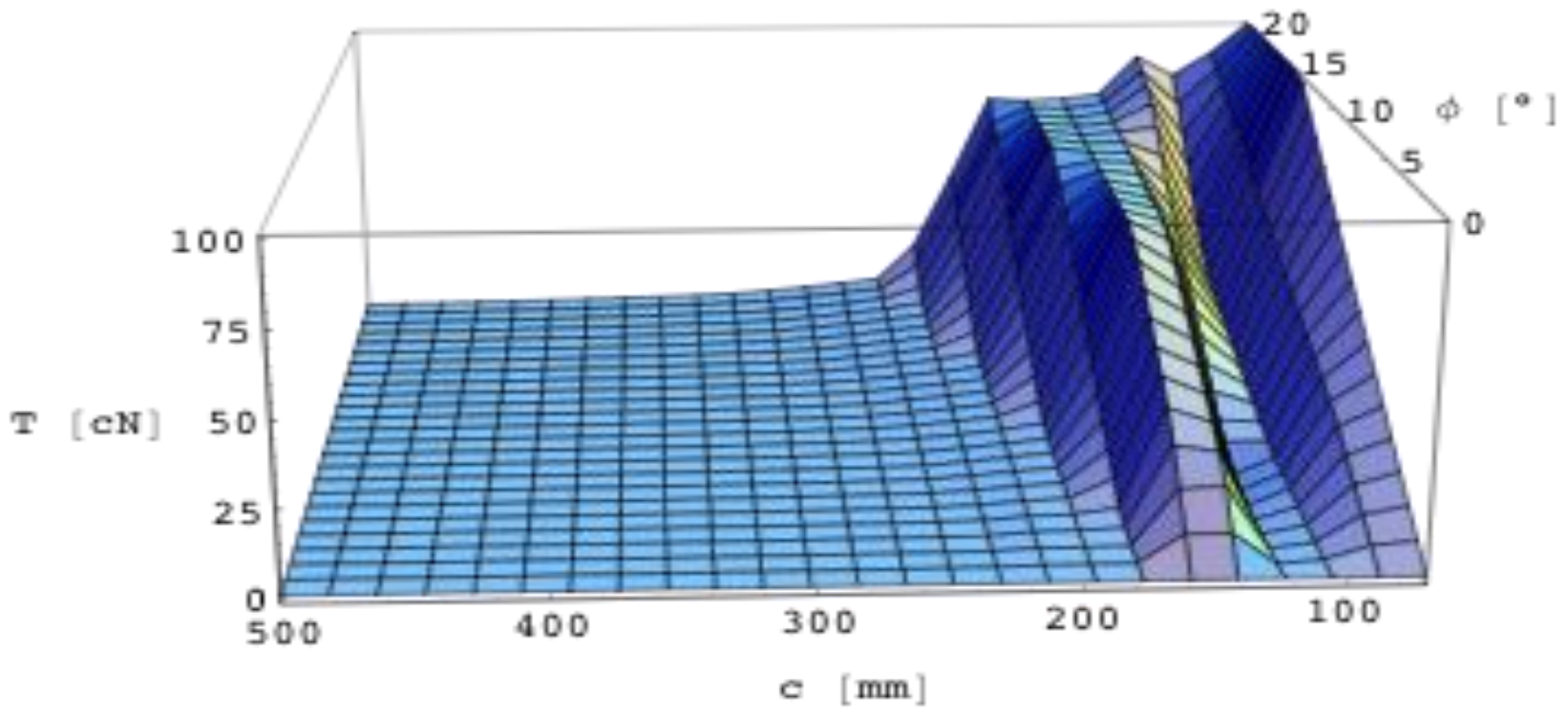

Fig. 6. Amplitude of oscillations as a function of the winding angle $\phi$ and the package radius $\mathrm{c}$ for constant unwinding velocity $V=2000 \mathrm{~m} / \mathrm{min}$ 
In Fig. 7 we show the amplitude of oscillations as a function of package radius and the unwinding velocity for a constant winding angle $\phi=5^{\circ}$. The curves of constant amplitude are simply straight lines, as expected, since at constant winding angle the amplitude depends only on the ratio $V / c$. This is a useful rule of thumb. We can opt for small package radii and reduce the unwinding speed, however for unwinding at large unwinding speeds it is necessary to increase the radius.



Fig. 7. Amplitude of oscillations as a function of package radius $c$ and the unwinding velocity $V$ for the winding angle of $\phi=5^{\circ}$

\subsection{Conic packages}

In Fig. 8 we show the tension during unwinding from conic packages of different apex angles. As expected on basis of Eq. (11), in the range of apex angles of conic packages, the effect of package shape is rather small.

\subsection{Packages with alternating parallely wound and cross wound layers}

It is possible to produce packages with mixed structure, for example packages with alternating layers of parallely and cross wound yarn. The package should be prepared so that the parallel wound layers are unwound when the lift-off point is moving backwards, and the interstitial cross wound layers are unwound when the liftoff point is moving forwards. This choice is motivated by the observation that cross wound packages lead to very high tensions when the yarn is unwound backwards. In addition, cross wound layers between two parallely wound layers are necessary to reduce the possibility of slips during unwinding. 
Pracek, S.; Sluga, F. \& Mozina,K.: Oscillations in the Yarn Tension During the Y...

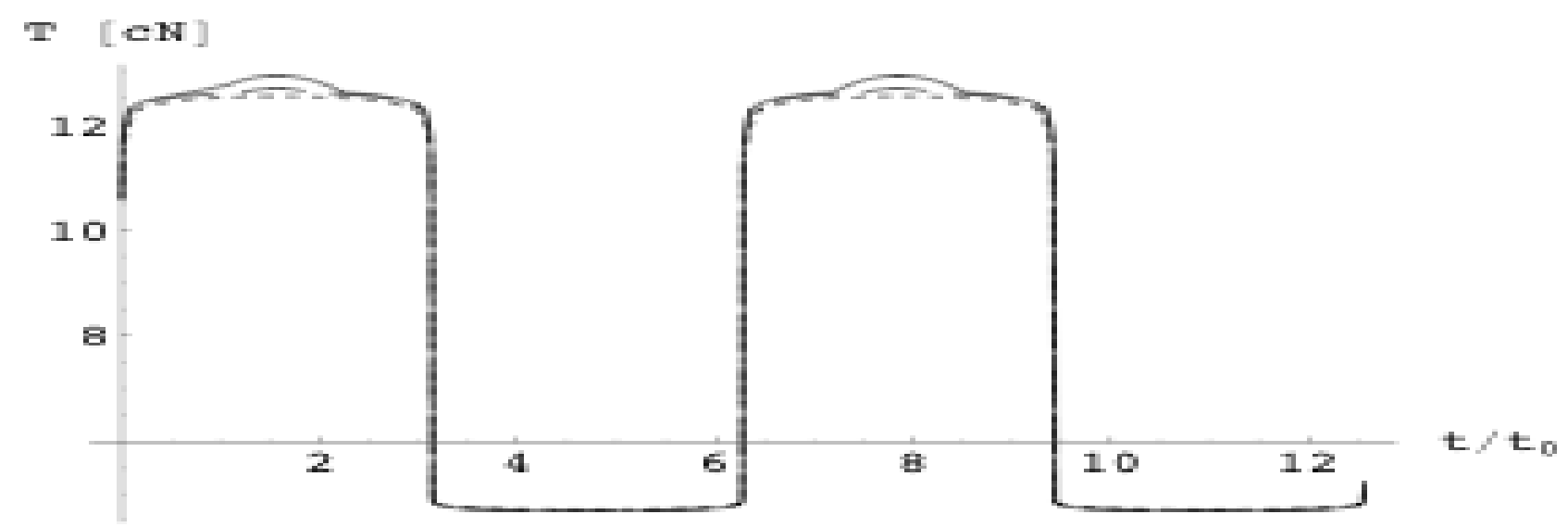

Fig. 8. Tension for conic packages of radius $c=200 \mathrm{~mm}$ and winding angle $\phi=10$ at unwinding velocity $V=2000 \mathrm{~m} / \mathrm{min}$ for different apex angles. $\alpha=0^{\circ}$ solid line, $\alpha=5^{\circ}$ dashed line, $\alpha=10^{\circ}$ dotted line

In Fig. 9 we compare a package with alternating layers with a regular cross wound package. The unwinding velocity is $2000 \mathrm{~m} / \mathrm{min}$, the package radius is 150 $\mathrm{mm}$ and the winding angle of cross wound layers is $10^{\circ}$. As expected, the parallely wound layers significantly reduce the tension that would otherwise possibly lead to yarn breaking. The alternating layer packages would therefore allow unwinding at greater unwinding velocities compared to conventional cross wound packages.

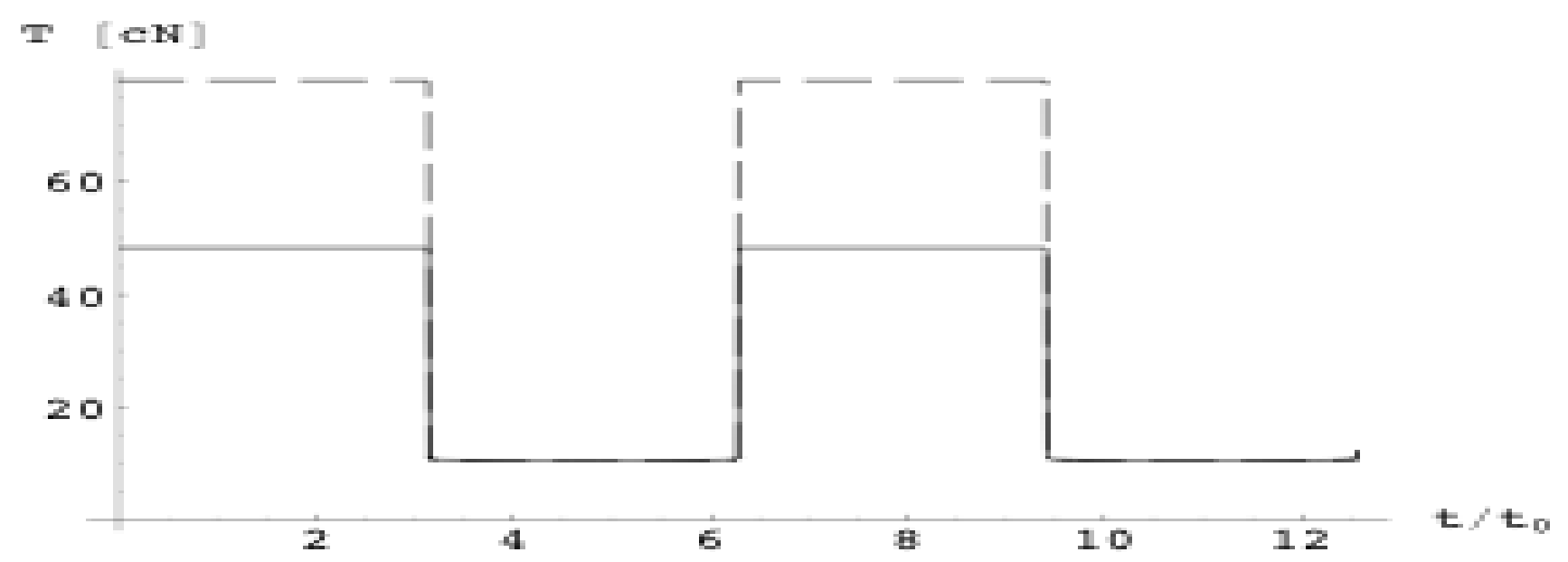

Fig. 9. Tension in packages with alternating layers (solid line) and in conventional cross wound packages (dashed line). Unwinding velocity $V=2000 \mathrm{~m} / \mathrm{min}$, package radius $c=150 \mathrm{~mm}$, winding angle $\phi=10^{\circ}$

In Fig. 10 we compare the amplitudes of the oscillations in the tension for both types of packages as a function of unwinding velocity and winding angle $\phi$ of cross would layers for package radius $c=150 \mathrm{~mm}$. From this plot we determine that in packages with alternating layers we can unwind even at very high winding angles. 


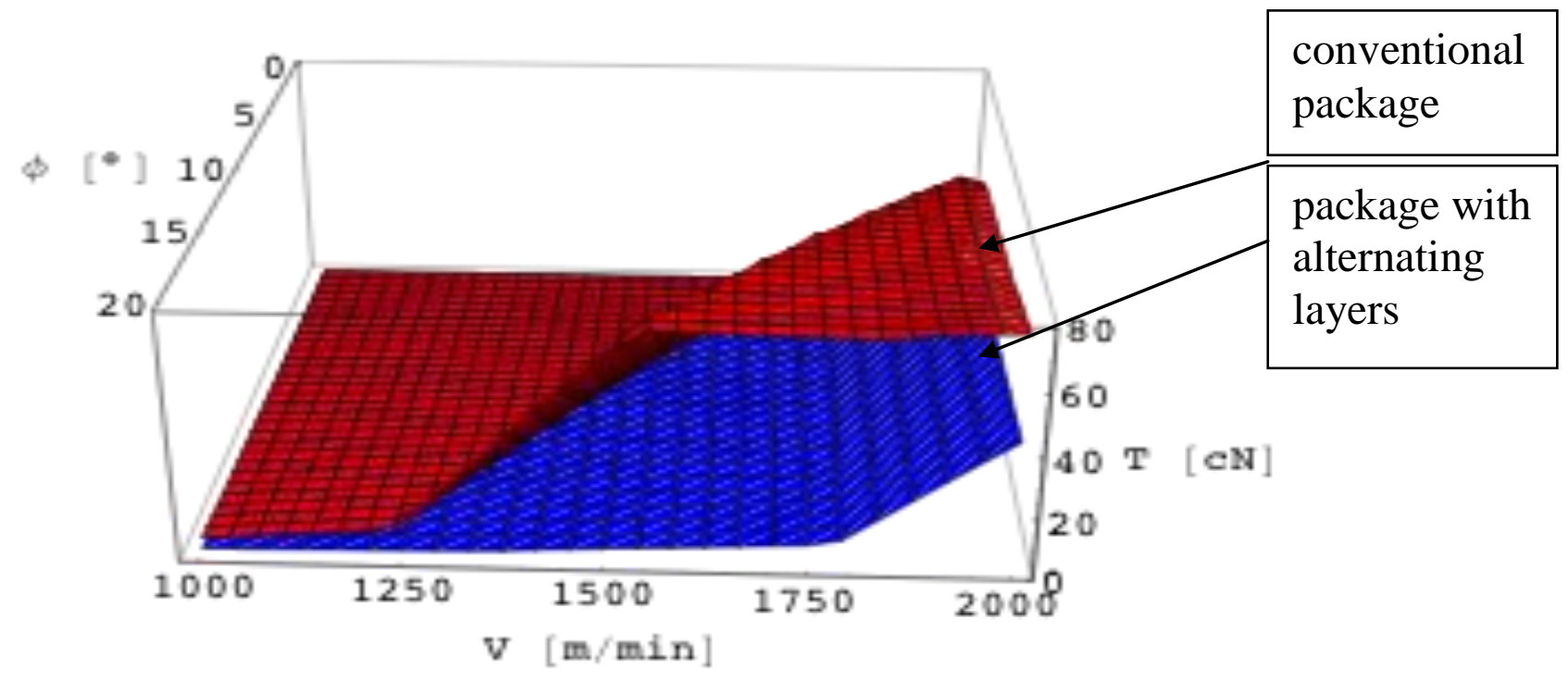

Fig. 10. Amplitude of the tension oscillations in packages with alternating layers and in conventional cross wound packages. Package radius is in both cases $c=150 \mathrm{~mm}$. In Fig. 11 we show the amplitude of tension oscillations in packages with alternating layers as a function of unwinding velocity and package radius. From the plot we can determine that at $V=2000 \mathrm{~m} / \mathrm{min}$ the package radius should be at least $150 \mathrm{~mm}$ if we want to avoid large oscillations of the tension. With high quality yarn the maximum allowable tension is higher, so it might be possible to unwind safely even from alternating layer packages with small radii, from $100 \mathrm{~mm}$ to $120 \mathrm{~mm}$

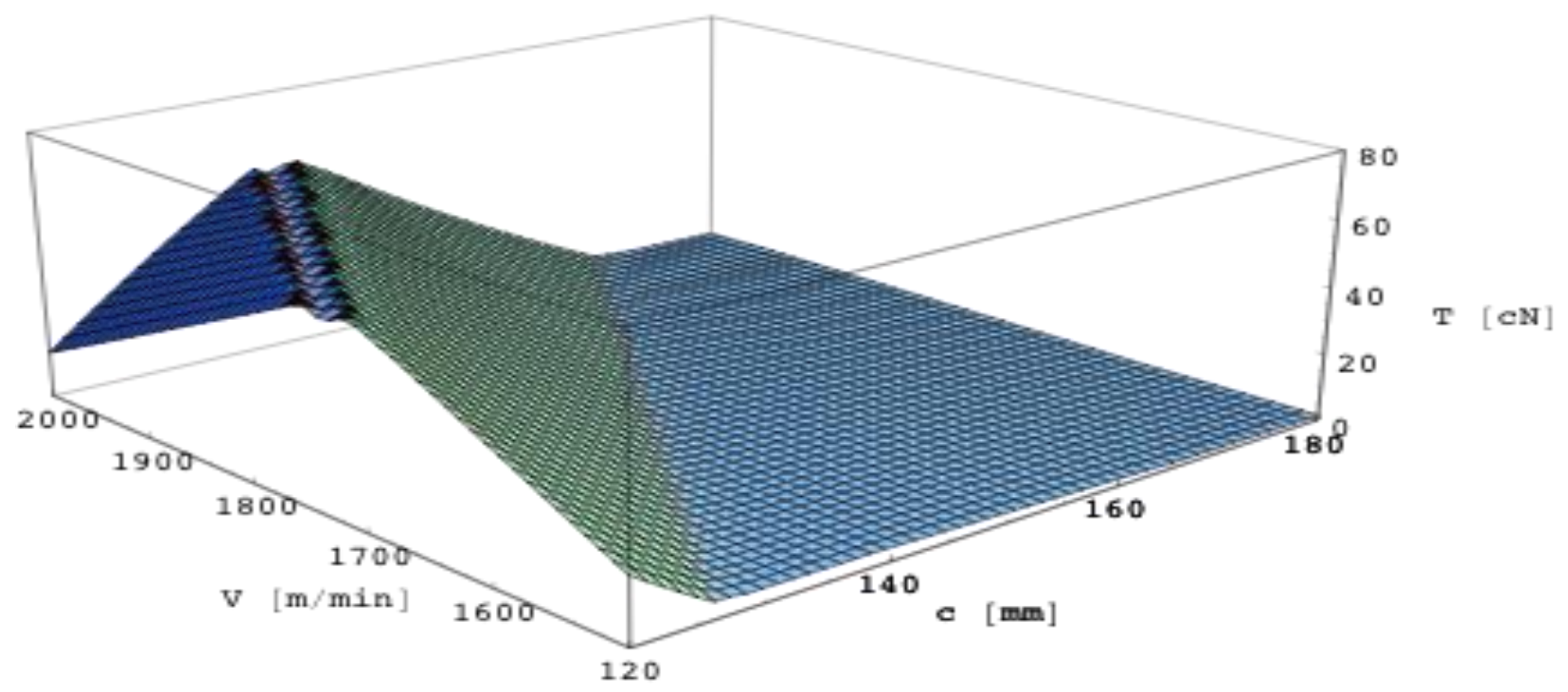

Fig. 11. Amplitude of the tension oscillations in packages with alternating layers. Winding angle $\phi=10^{\circ}$

\section{Conclusions}

We have shown that a combination of theoretical modelling and empirically determined relation between the yarn tension and the angular velocity of the yarn in the balloon can be used to fruition in determining a suitable set of parameters for 
Pracek, S.; Sluga, F. \& Mozina,K.: Oscillations in the Yarn Tension During the Y...

unwinding from packages of various designs. We have reached the following conclusions:

- In cross-wound packages, when the yarn unwinds backwards the angular velocity is higher as when the yarn unwinds forwards, which leads to oscillations in the yarn tension. Abrupt changes of tension occur at the edges of the package.

- Unwinding at high velocity $(2000 \mathrm{~m} / \mathrm{min})$ from regular cross-wound packages is impossible for package radii below $150 \mathrm{~mm}$.

- Oscillations of tension are smaller in packages with small winding angle (parallel-wound packages), but for such packages yarn slips can occur during unwinding.

- Alternatively, the oscillations of tension can be reduced if only those layers of yarn that are unwound backwards are parallely wound.

Using packages with alternating layers of parallely and cross wound yarn, both the maximum tension and the amplitude of the tension oscillations can be significantly reduced. In this case it is possible to safely unwind from packages of smaller radius even at higher unwinding velocities. This would allow higher production rates without increased down-time due to yarn breaking.

We have determined that it is possible to safely unwind yarn from packages with alternating layers of radius of $150 \mathrm{~mm}$ and in some circumstances even 120 or 100 $\mathrm{mm}$.

\section{References}

Barr, A. E. D., Catling, H. (1976). Manual of Cotton Spimmimg, Volume Five. Butterworth

Fraser, W. B., Ghosh, T. K., Batra, S. K. (1992). On unwinding yarn from cylindrical package. Proc. R. Soc. Lond. A, 436 479-498.

Fraser, W. B., (1992). The effect of yarn elasticity on an unwinding ballon. J. Tex. Inst, 83 603-613

J.D.Clark, W.B.Fraser, R. Sharma and C.D.Rahn. (1998). The dynamic response of a ballooning yarn:theory and experiment. Proc. R. Soc. Lond. A, 454 2767-2789

J.D.Clark, W.B.Fraser, D.M.Stump. (2001). Modelling of tension in yarn package unwinding. J.Engi.Mathema., 40, 59-75

Kothari,V.K.,Leaf G.A.V. (1979). The unwinding of yarns from packages, PartII: The theory of yarn-unwinding.J.Text.Inst 70 (3)95-105

Padfield, D. G. (1958). The Motion and Tension of an Unwinding Thread. Proc. $R$. Soc., vol. A245, 382-407

Praček, S. (2002). Modification of yarn unwinding dynamics. Dissertation, University of Ljubljana

Praček,S. (2007). Theory of string motion in the textile process of yarn unwinding. Int. J.Non.S.Num.S. 8 (3)451-460

Roberson, J. A., Crowe, C. T. (1980) Engineering fluid dynamics. Houghton Mifflin Company, Boston, second edition 